\title{
Enhanced and Constant-value Transient Diffraction Efficiency from a Recorded Grating in a $\mathrm{BaTiO}_{3}$ Crystal
}

\author{
S. M. Sharif ${ }^{1}$ and K. Ogusu ${ }^{2}$ \\ ${ }^{1}$ Department of Physics, Shah Jalal University of Science and Technology, Sylhet-3114, Bangladesh \\ ${ }^{2}$ Department of Electrical and Electronic Engineering, Shizuoka University, 3-5-1, Johoku, \\ Hamamatsu 432-8561, Japan
}

Received 22 July 2010, accepted in final revised form 30 December 2010

\begin{abstract}
A simple method has been proposed here to get an enhanced and constant-value transient diffraction efficiency for a large time scale from a recorded grating in an undoped $\mathrm{BaTiO}_{3}$ crystal. After writing a steady-state grating by two cw recording beams $(\lambda=514.5 \mathrm{~nm})$, when the recording beams are switched off, the recorded grating as well as the diffraction efficiency decreases at a faster rate initially, and thereafter reaches to almost a stationary level for some few seconds. The diffraction efficiency for this level is very small compared to that for a steady-state grating and it becomes smaller slowly with time. We can increase (about twice the initial stationary-level value) this small diffraction efficiency from its any initial value and have this value (increased) constantly for a large time scale by using a suitable backward-pulsed reading beam containing the same properties as the recording beams.
\end{abstract}

Keywords: Photorefractive effect; Transient diffraction efficiency; Steady-state volume grating; Ferroelectric perovskite crystal.

(c) 2011 JSR Publications. ISSN: 2070-0237 (Print); 2070-0245 (Online). All rights reserved.

doi:10.3329/jsr.v3i2.5593 J. Sci. Res. 3 (2), 217-224 (2011)

\section{Introduction}

Researches with photorefractive materials have become a wide and active area of research during the past several years, due to their potential applications in various fields of optics. $\mathrm{BaTiO}_{3}$ is an attractive photorefractive material because of its large electrooptic coefficient that lead to strong coupling for two- and four-wave mixing. High value of diffraction efficiency (i.e., the fraction of the power of the incident beam transferred into the diffracted beam passing through a medium [1]) as well as phase-conjugate reflectivity is always desired for their different applications. Researches are going on to investigate and increase the diffraction efficiency from a recorded grating inside a photorefractive crystal [2-7]. Yue et al. [8] observed a unique increase in transient diffraction efficiency

\footnotetext{
${ }^{1}$ Corresponding author:sharif-phy@sust.edu
} 
(TDF) which was up to ten times greater than the stationary TDE and was caused by the preillumination of $\mathrm{Bi}_{4} \mathrm{Ti}_{3} \mathrm{O}_{12}$ at a low temperature. Smirl et al. [9] observed in $\mathrm{BaTiO}_{3}$ an increase of the holographic diffraction efficiency in the dark after holographic writing with ps laser pulses. This effect can be understood in terms of the two-center charge transport model [10]. In our previous works, we experimentally observed a unique increase in the TDE for a given pulsed reading beam under suitable conditions in an undoped $\mathrm{BaTiO}_{3}$ crystal at room temperature [11]. Using this increased TDE we could also observe a unique increase in the switched phase-conjugate reflectivity (SPCR) [12, 13]. The observations were attributed to the presence of two shallow levels in the charge transport process in the crystal even at room temperature in our proposed model [11-15].

In all of our previous works, we observed that after writing a steady-state grating by two $\mathrm{Cw}$ recording beams ( $\lambda=514.5 \mathrm{~nm}$ ), when the recording beams are switched off, the recorded grating as well as the diffraction efficiency decreases at a faster rate initially, and thereafter reaches to a nearly steady-state level for a short time scale (i.e., it remains almost constant for some few seconds). The diffraction efficiency for this level is very small with respect to that for a steady-state grating and it becomes smaller slowly as time goes on (i.e., for a large time scale). Our previously observed [11-15] unique enhancement of TDE or SPCR was from this short time nearly steady-state level and the investigations were for maximizing their transient values. The enhanced value of TDE or SPCR was for a given pulse of the backward reading beam and could somewhat be controlled by using proper interacting beams.

For a recorded grating, one major problem is the degradation of recorded gratings with time (even in dark) due to the recombination of the charge carriers, and also by the reading beam during the read-out process. Therefore, the diffraction efficiency (hence, the ratio between diffracted and incident light intensity of the probe beam) from a recorded grating decreases with time whether a reading beam is present or not. However, in the presence of a reading beam, the diffraction efficiency decreases with increasing reading beam (or pump beam) power [3, 7, 11] (although, the TDE is seen to be increased for some initial pulses when a pulsed reading beam is incident on the grating $[11,12]$ ). Despite the degradation of the recorded grating, if an enhanced and constant-value of TDE for a large time scale (e.g., 10-30 mins.) is possible ( from its any initial value) from a recorded grating, after a significantly later time of recording, then it would in principle be useful in various applications such as optical image processing, holographic storage etc.

In this study, we give a method to get an enhanced (about twice the stationary-level value) and also a constant value of TDE for a large time scale, in an undoped $\mathrm{BaTiO}_{3}$ crystal at room temperature, which can be of practical use. To our knowledge, a report on the observation of such a constant-value TDE for a large time scale has not yet been reported. The present observation can also be explained with our previously proposed model of the charge transport process.

\section{Experiment}

Fig. 1 shows an experimental setup where the interacting beams (i.e., the recording and reading beams) from an $\mathrm{Ar}^{+}$laser were set as in our previous experiments (i.e., degenerate 
four-wave mixing geometry) [11-13]. The recording beams and the reading beam are arranged conventionally from an unexpended light beam of a single longitudinal-mode $\operatorname{Ar}^{+}$laser $(\lambda=514.5 \mathrm{~nm}$, beam diameter $=1.2 \mathrm{~mm})$. The polarization of all the interacting beams is extraordinary. An undoped $\mathrm{BaTiO}_{3}$ crystal $\left(5 \times 5 \times 2 \mathrm{~mm}^{3}, 0^{\circ}\right.$ cut $)$ is used as our sample whose absorption constants are $\alpha_{0}=2.79 \mathrm{~cm}^{-1}$ and $\alpha_{e}=2.33 \mathrm{~cm}^{-1}$ at the operating wavelength. The orientation of the crystal and the interacting beams were set so that the angle $\theta$ between the grating wave vector $\boldsymbol{K}$ and the crystal c-axis was as large as possible (here $\theta=16^{\circ}$ ) without using any matching medium, to ensure a large contribution of the electro-optic coefficient $\mathrm{r}_{42}$.

We first record a steady-state transmission grating by two extraordinarily polarized recording beams while keeping the reading beam switched off. An ordinarily polarized weak He-Ne-laser cw probe beam $(\lambda=632.8 \mathrm{~nm})$ enters the crystal simultaneously at the Bragg angle and the diffracted beam from the recorded grating is monitored by a

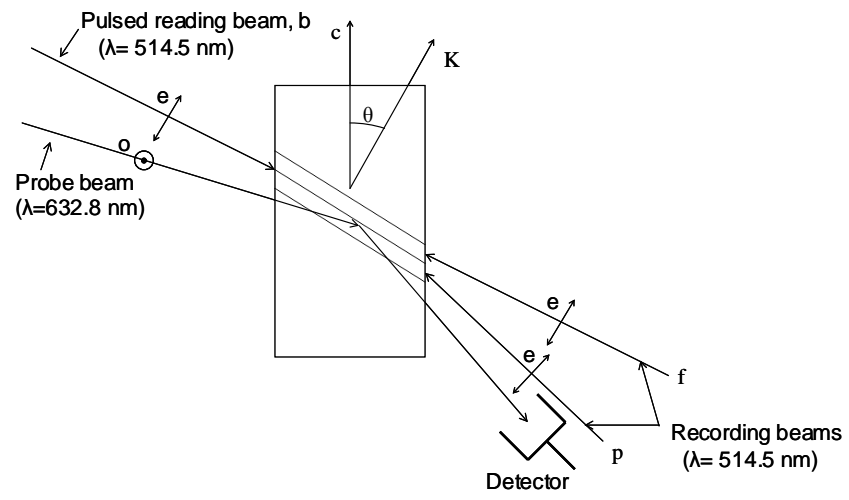

Fig. 1. Four-wave mixing configuration used in the experiment. The two recording and the reading beams $(\lambda=514.5 \mathrm{~nm})$ have extraordinary polarization, and the weak probe beam $(\lambda=632.8 \mathrm{~nm})$ has ordinary polarization.

photodiode behind the crystal. The intensity of the probe beam is kept less than $100 \mu \mathrm{W} / \mathrm{cm}^{2}$ in order to avoid its influence on the recorded grating. Fig. 2 shows the temporal variation of the diffracted probe beam power during the recording and reading process. A pulsed backward-pump beam ( $\lambda=514.5 \mathrm{~nm}$ and extraordinarily polarized) with a pulse width of $\sim 1$ sec and a period of $T$ is then incident at $t=0$ after switching off the recording beams at $t=-20 \mathrm{sec}$. During the recording process the diffracted beam power reaches to a steady-state value within about 200 sec. When the recording beams are switched off, the diffracted beam power decreases sharply, and thereafter reaches to a nearly steady-state level which remains almost constant for some few seconds. The diffraction efficiency for this level is very small compared to that for a steady-state grating and it becomes smaller slowly as time goes on (i.e., for a larger time scale). 


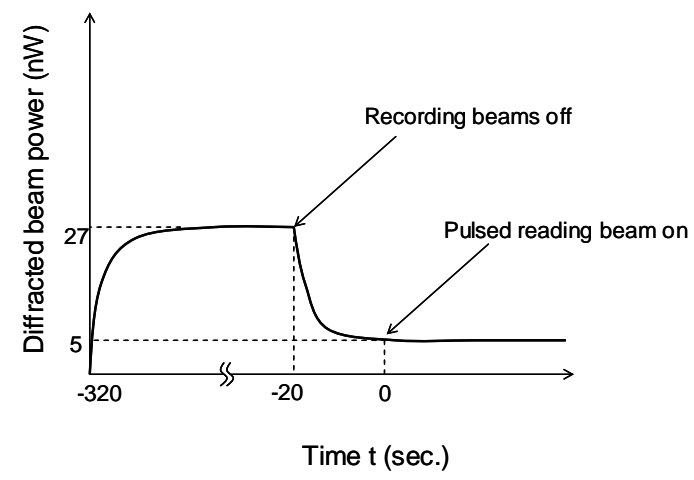

Fig. 2. Temporal variation of the diffracted beam power of a weak probe beam $(\lambda=632.8 \mathrm{~nm})$ entering the crystal at the Bragg angle.

\section{Results and Discussion}

Fig. 3 shows the temporal variation of the diffracted probe beam power for various reading beam powers $P_{\mathrm{b}}$ and time periods $T$ (when it is in pulsed mode). As we observed in our previous experiments $[11,12]$ that the TDE or SPCR can be affected by the used values of the interacting beam ratio, $r_{\text {int }}\left(=\mathrm{P}_{\mathrm{b}} /\left(\mathrm{P}_{\mathrm{f}}+\mathrm{P}_{\mathrm{p}}\right)\right)$ and the recording beam ratio, $r_{\text {rec }}$ $\left(=P_{p} / P_{f}\right)$, where $P_{i}(i=b, f$ and $p)$ represents the power of each beam denoted in Fig. 1 , we have chosen here the values for obtaining maximum diffraction efficiency. For a cw reading beam, the diffracted beam power (solid line) decreases gradually with time due to the erasing effect of the recorded grating by the reading beam. Here the recording beam

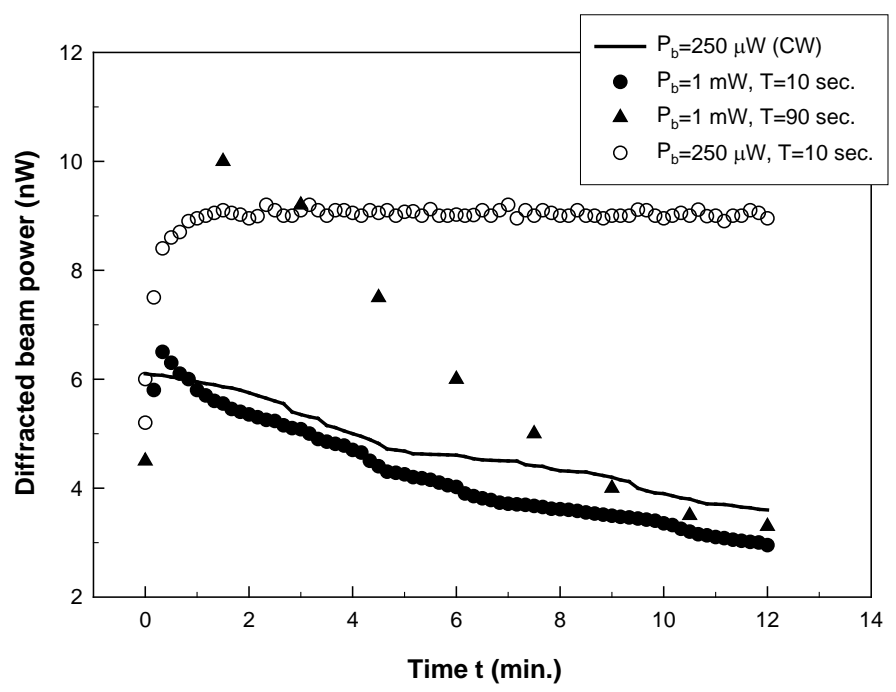

Fig. 3. Temporal variation of the diffracted probe beam power for $\mathrm{cw}$ and pulsed reading beam with powers $P_{\mathrm{b}}$ and time periods $T$. 
intensity and the reading beam intensity are $7 \mathrm{~mW} / \mathrm{cm}^{2}$ and $44 \mathrm{~mW} / \mathrm{cm}^{2}$, respectively and $r_{\text {rec }}=0.1$. For a pulsed reading beam of power $1 \mathrm{~mW}$ (for $T=90$ and $10 \mathrm{sec}$.) the diffracted beam power as well as the TDE grows and reaches a peak value at a certain initial pulse of the reading beam (i.e., at second pulse for $T=90 \mathrm{sec}$ (triangle) and at third pulse for $T=10 \mathrm{sec}$ (solid circle)) after switching on at $t=0$. After the peak, the TDE decreases gradually with time for further incident pulses of the reading beam. Here the intensity of the reading beam is four times greater than that of the $\mathrm{cw}$ reading beam. The observed result for $T=90 \mathrm{sec}$ is similar to our previous experimental works, where the investigations were for maximizing the diffraction efficiency. However, for a pulsed reading beam with the same intensity of the $\mathrm{cw}$ reading beam and pulse period of $T=10$ sec, the TDE reaches a constant value within a few pulses of the reading beam. It is surprisingly observed that in spite of erasing of the recorded grating by the incidence of reading beam pulses, the TDE remains constant for a large time scale (i.e., up to 20 mins. or more). Fig. 4 shows the temporal variation of the diffracted probe beam power for different $\mathrm{cw}$ and pulsed reading beam powers $P_{\mathrm{b}}$ and time periods $T$ (when it is in pulsed mode). For a $\mathrm{cw}$ reading beam the diffracted beam power as well as the diffraction
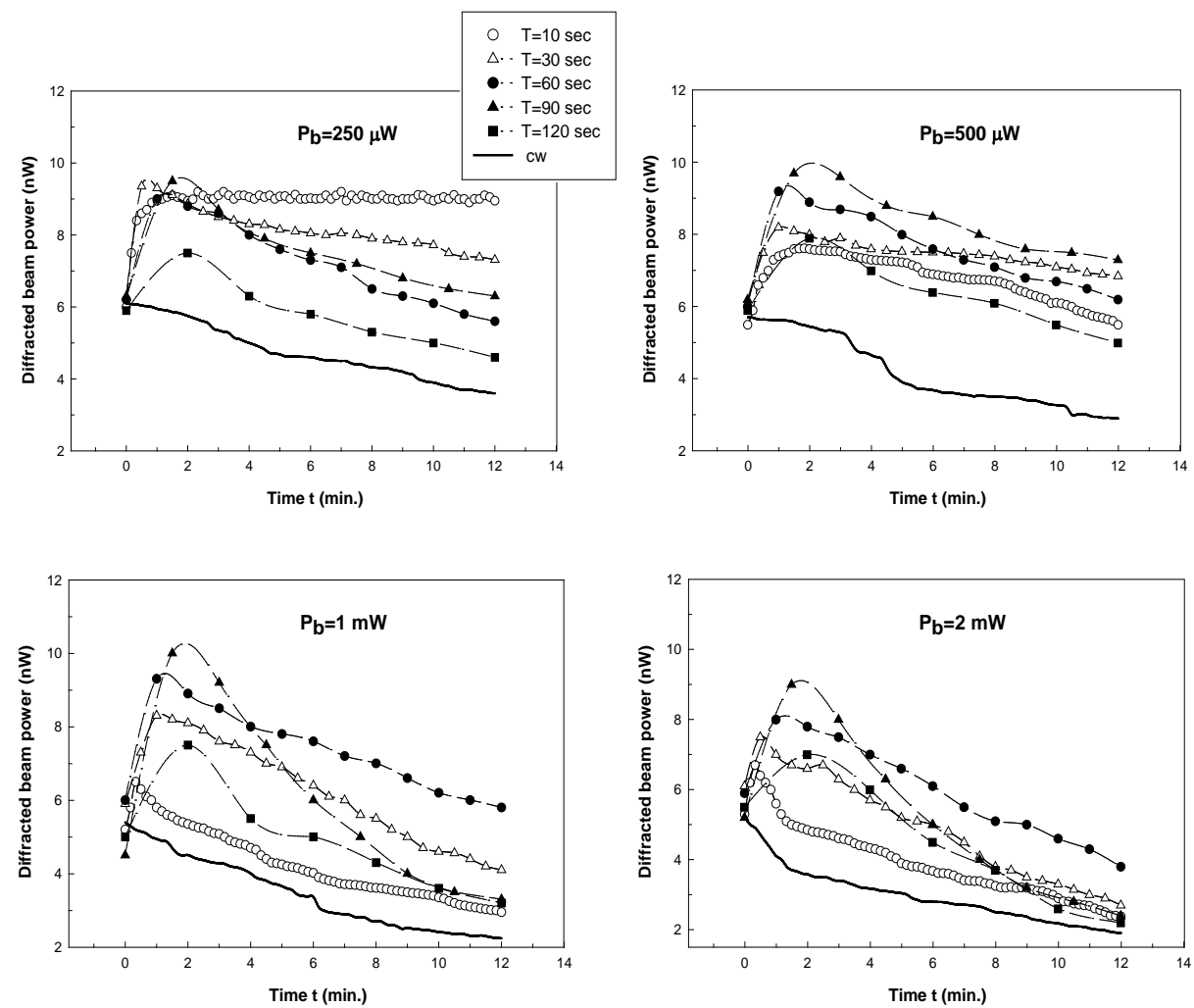

Fig. 4. Temporal variation of the diffracted probe beam power for different cw and pulsed reading beam powers $P_{\mathrm{b}}$ and time periods $T$. The curves drawn here are only guides for the eye. 
efficiency decreases with increasing the reading beam power $\mathrm{P}_{\mathrm{b}}$. For the pulsed reading beams with different pulse periods $T$ (i.e., $T=10$, 30, 60, 90 and $120 \mathrm{sec}$.), the diffraction efficiency reaches to a peak then decreases with time (except for the pulsed reading beam with $P_{\mathrm{b}}=250 \mu \mathrm{W}$ and $T=10$ sec.). For the pulsed reading beam of power $P_{\mathrm{b}}=250 \mu \mathrm{W}$ and of $T=10 \mathrm{sec}$. the diffraction efficiency remains constant for a large time scale.

The observed result of this constant-value TDE can also be explained with our previously proposed charge transport model [11, 12]. In the model, we consider two shallow centers $L_{1}$ and $L_{2}$ along with one deep center $L_{\mathrm{D}}$, as shown in Fig. 5 . The deep center involves both positive and negative charge carriers, while the shallow centers involve only positive charge carriers. We also assume that the photoexcitation crosssection of $L_{1}$ is larger than that of $L_{2}$ (i.e., $s_{1}>s_{2}$ ) and the thermal excitation coefficient of $L_{2}$ is larger than that of $L_{1}$ (i.e., $\beta_{2}>\beta_{1}$ ). Due to the intensity distribution, charge gratings are formed in both the shallow and deep centers and can reach a steady-state form for a sufficiently long recording time. For the incidence of a reading beam (either cw or pulse), these gratings might be erased slightly or largely depending on its intensity and irradiant duration. During the illumination of a reading pulse, additional holes (as holes are believed to be the dominant charge carriers in $\left.\mathrm{BaTiO}_{3}[10,16,17]\right)$ are photoexcited from $L_{\mathrm{D}}$ and most of them are accumulated to $L_{2}$ through the valence band and $L_{1}$. When the pulse is over, holes are thermally excited from $L_{2}$ and accumulated at a large rate to $L_{1}$ (since $\beta_{2}>\beta_{1}$ ) and finally to $L_{D}$. Therefore, the concentration of holes in $L_{1}$ increases in the dark (i.e., while the reading beam is in off-state) for a while. If the dark-time is large enough, holes are recombined to $L_{\mathrm{D}}$ from $L_{1}$ through the valence band. Photoexcitation from $L_{\mathrm{D}}$ is also largely dependent on the intensity of the reading beam pulse. A high intensity of the reading beam pulse can photoexcite large charge carriers, however, it erases the recorded grating largely.



Fig. 5. Charge transport model with deep level $L_{\mathrm{D}}$ and two shallow levels $L_{1}$ and $L_{2}$. Arrows indicate excitation and recombination of charge carriers in valence and conduction bands.

For the incidence of a reading pulse, an instantaneous grating is formed by the reading and the diffracted grating which is added with the previously recorded grating. Therefore, the grating depth can be maximized when $L_{1}$ has maximum charge concentration. For the case of constant value TDE, with a moderate reading beam pulse 
power as observed in Fig. 3, the erased grating due to the reading pulse is compensated by the instantaneous grating formed by the reading and the diffracted beam pulses, and it is due to this charge transport process that the grating depth remains constant for such a large time scale. For the other cases of reading beam power, the erasing effects are dominant and the TDE decreases with time (except for some initial pulses of the reading beam power of $1 \mathrm{~mW}$ with pulse period $T=90 \mathrm{sec}$ and $T=10 \mathrm{sec}$; and hence the growing of TDE during those pulses are because the resultant grating suppresses the previously present grating before the incidence of pulses). The pulse width used ( 1 sec) throughout the experiment is the same one used in our previous experiments.

We also observed the same result of constant value of TDE even if the pulsed reading beam is incident at a significantly later time (e.g., after writing a steady-state grating, if the recording beams are switched off at $t=-(10-20)$ mins. in Fig. 2) after switching off the recording beam. However, the enhancement of TDE is found to slightly vary inversely to the intermediate time between when the recording beams are switched-off and the reading beam is switched-on. For $t=-20 \mathrm{sec}$, TDE increases to about twice the value with respect to that when no reading pulses are incident on the crystal.

\section{Conclusions}

For a recorded grating, one major problem is the degradation of recorded gratings as well as the diffraction efficiency with time whether a reading beam is present (during the readout process) or not. In this paper, a simple method has been reported to get enhanced and constant-value transient diffraction efficiency (from any of its initial value) for a large time scale, in spite of degradation of the recorded gratings during the read-out process. A suitable pulsed backward reading beam containing the same properties as the recording beams can give this enhanced constant-value transient diffraction efficiency from a recorded grating (even after a significantly later time of recording), which could in principle be useful in many different applications such as optical image processing, phase conjugation, holographic storage etc.

\section{References}

1. P. Yeh, Introduction to Photorefractive Nonlinear Optics (Wiley, 1993).

2. Y. Takayama, A. Okamoto, K. Sato, J. Opt. Soc. Am. B 14, 2570 (1997). doi:10.1364/JOSAB.14.002570

3. Y. Takayama, A. Okamoto, K. Sato, Opt. Commun. 123, 603 (1996). doi:10.1016/0030-4018(95)00584-6

4. K. Buse, J. Frejlich, G. Kuper, E. Krätzig, Appl. Phys. A 57, 437 (1993). doi:10.1007/BF00331783

5. F. Rickermann, S. Riehemann, K. Buse, D. Dirksen, G. von Bally, J. Opt. Soc. Am. B 13, 2299 (1996). doi:10.1364/JOSAB.13.002299

6. I. A. Taj, T. Mishima, J. Opt. Soc. Am. B 16, 924 (1999). doi:10.1364/JOSAB.16.000924

7. I. A. Taj, P. Xie, T. Mishima, J. Opt. Soc. Am. B 17, 1740 (2000). doi:10.1364/JOSAB.17.001740

8. X. Yue, K. Buse, F. Mersch, E. Krätzig, R. A. Rupp, J. Opt. Soc. Am. B 15, 142 (1998). 
doi:10.1364/JOSAB.15.000142

9. A. L. Smirl, K. Bohnert, G. C. Valley, R. A. Mullen, T. F. Boggess, J. Opt. Soc. Am. B 6, 606 (1989). doi:10.1364/JOSAB.6.000606

10. P. Tayebati, D. Mahgerefteh, J. Opt. Soc. Am. B 8, 1053 (1991). doi:10.1364/JOSAB.8.001053

11. S. M. Sharif and K. Ogusu, Jpn. J. Appl. Phys. 43, 6166 (2004). doi:10.1143/JJAP.43.6166

12. S. M. Sharif and K. Ogusu, Opt. Mat. 27, 67 (2004). doi:10.1016/j.optmat.2004.02.016

13. S. M. Sharif and K. Ogusu, CLEO/Pacific Rim 1, 28 (2003).

14. M. H. Rashid, Y. Haque, S. K. Das, S. M. Sharif, SUST Studies 8, 97 (2007).

15. M. H. Rashid, Y. Haque, S. K. Das, S. M. Sharif, SUST Studies 12, 106 (2010).

16. G. A. Brost, R. A. Motes, J. R. Rotge, J. Opt. Soc. Am. B 5, 1879 (1988). doi:10.1364/JOSAB.5.001879

17. P. Günter, J. P. Huignard (Eds.), Photorefractive Materials and their Applications II, vol. 62, (Springer-Verlag, 1988). 